\title{
Residual Stress Buildup in Ti Components Produced by Cold Spray Additive Manufacturing (CSAM)
}

\author{
V. Luzin ${ }^{1}$ O. Kirstein ${ }^{2}$ S. H. Zahiri ${ }^{3}$ D. Fraser $^{3}$
}

Submitted: 15 November 2019/in revised form: 30 April 2020/Published online: 20 May 2020

(C) ASM International 2020

\begin{abstract}
Cold spray has been developed recently to be used as an additive manufacturing technology in order to fabricate bulk components. Residual stresses are known to build up in coatings made by cold spray; therefore, cold spray additive manufacturing (CSAM) is also expected to generate residual stress in bulk parts and components, and that residual stress can lead to shape distortions or component cracking. The residual stress analysis has been applied to some generic sample shapes, a thick patch deposit and a vertical wall, produced by CSAM out of Ti powder. The residual stress mapping has been achieved using neutron diffraction technique and analyzed within a modeling approach. The analysis allowed it to be determined as to what were the major contributions into the overall stress field and to establish the main sources of the residual stress, providing an analytical tool for prediction of the residual stress buildup in more complex shapes.
\end{abstract}

Keywords additive manufacturing · cold spray · processing $\cdot$ properties $\cdot$ residual stress

V. Luzin

vladimir.luzin@ansto.gov.au

1 Australian Nuclear Science and Technology Organisation, Lucas Heights, NSW 2232, Australia

2 European Spallation Source ESS, 22363 Lund, Sweden

3 Commonwealth Scientific and Industrial Research Organisation, Clayton, Melbourne, VIC 3168, Australia

\section{Introduction}

The majority of cold spray applications and research studies in the recent years have been focused mostly on production of coatings of different kinds, usually on flat or cylindrical surfaces (Ref 1). Especially when coatings are thin, such as in many corrosion protection applications, the extension into more complex surfaces is not extremely challenging and the approach is very similar to surface painting, though in this case with metal "paint," metallization. More problems and challenges occur when thick deposits and complex 3D shapes are under consideration. Probably due to this, much lesser attention is paid to adopting the spraying technique to less trivial applications that can be called cold spray additive manufacturing (CSAM) (Ref 2-4). Among such applications are (1) the surface repair and dimensional tolerance restoration that deals with addition of material to the existing part instead of partial loss of the original material and (2) production of complex 3D parts by spraying that are fully made of the sprayed material. In both cases, the technological challenges are great because these require development of complicated robotic systems with strong support from the basic understanding of the cold spray process with multiple and intertwined processing parameters. These include gas pressure and temperature, nozzle geometry, powder type and characteristics, feed rate, traverse speed, traverse trajectory, spray angle, etc. As a result, all the above affect the properties of the final product, which are supposed to be optimized for specific applications. Also, depending on application, the most important properties can be fatigue properties, hardness, mechanical strength, porosity, ductility, machinability and it occurs that the residual stress is one of the characteristics that is intertwined with the above mechanical properties and it is directly related to the 
structural integrity and overall performance of the products made by cold spray.

The topic of residual stress is not new in CS research. However, previously, the studies of the (residual) stress analysis were focused on different scale levels and briefly can be categorized as following.

(1) A splat scale (that can be called microscopic level) was in the focus due to fundamental problem of the splat bonding and deposit forming mechanism reflected in many seminal studies (Ref 5-7), just to cite few. The residual stress, as well as the related yielding, plastic deformation phenomena, were integral part of the studies since it was essential for understanding of the cold spray deposition mechanism. This microscopic level of understanding is expressed in numerous single-splat deposition simulations (Ref 8, 9). Such simulations, especially multi-particle ones (Ref 10, 11), show that the residual stress pattern development within splats is very complex temporally and spatially; the stress develops due to complex interaction of temperatureand time-dependant localized plasticity that can compete with stress relaxation mechanism due to temperature effects and/or defect structures. Overall, for multiple-particle deposits, the residual stress on microscopic level is highly oscillating reaching locally the yield point of material (Ref 8, 9, 12).

(2) In the scale of thin coatings (that can be called mesoscopic), the average (coherent) effect of stress distribution results in the concept of the deposition stress, the stress that is associated with an infinitesimal thin layer of material, which, nevertheless, contains many particles. Averaged over the ensemble of many particles, all localized oscillations disappear giving some moderate value of deposition stress. This concept of deposition stress is the basis for many, if not all, analytical deposition models (e.g., Ref 13, 14), as well as experimental studies (Ref 12). It is usually accepted and frequently stated that deposition stress in CS coatings is compressive (Ref 15).

(3) In the scale of large deposits manufactured by CS (that can be called macroscopic), stress can have complex three-dimensional distribution, depending on shape, deposition strategy, sizes, etc. Although on the mesoscopic level the same deposition stress at work, when this concept is applied for 3D objects made by CSAM, the role of the deposition stress might be much obscured. This can be illustrated with a simple example. While the residual stress and the deposition stress are essentially the same for thin coatings (this is the basis of the Stoney's approach
(Ref 16), for multilayered deposition of thick coatings the residual stress and the deposition stress are not the same. Although deposition stress can be the same for each individual layer, the residual stress is not constant, it has through-thickness distribution and even sign of the residual stress might be different for different layers. Obviously, going from the one-dimensional system of (infinite) coatings to arbitrary three-dimensional objects or arbitrary shapes and sizes can be challenging conceptually and experimentally.

While the formation of residual stress in CS coatings (on flat and cylindrical surfaces) is more or less understood (Ref 12, 14, 17), the amount of the experimental data on residual stress analysis in 3D parts is very limited. However, applying combined knowledge of residual stress formation in coatings and principles of stress distribution in 3D objects, some general predictions can be made in cases of not very complicated shapes/geometries. The goal of the present paper is to make bridge from the meso- to macroscopic scale and to demonstrate how concept of the deposition stress developed for coatings can be applied for samples of more general geometries.

\section{Experimental}

\section{Samples: Materials and Spraying Conditions and Characterization}

A commercially available CP titanium powder of grade 4 was used to spray samples for this study. The average particle size was $27 \mu \mathrm{m}$, while the powder size distribution and particle morphology are presented in Fig. 1(a).

A cold spray system (CGT KINETIKS 3000) with converging/diverging (de Laval) nozzle was used for direct fabrication of the $\mathrm{Ti}$ structures for this study. The nozzle geometries were $51.2 \mathrm{~mm}$ converging section, $2.7 \mathrm{~mm}$ throat diameter, $70.3 \mathrm{~mm}$ diverging section and $8.3 \mathrm{~mm}$ exit diameter. The nozzle was made of tungsten carbide. The deposition was carried out at 24 bars pressure and $800{ }^{\circ} \mathrm{C}$ spray temperature, with $\mathrm{N}_{2}$ as carrier gas. These parameters were chosen in relation to maximum capacity of the CGT KINETIKS 3000 cold spray system and in order to achieve the maximum particle velocity needed for deposition of a dense and low porosity material. The nozzle was mounted on a robotic arm to precisely control the cold spray supersonic jet motion during deposition. The transverse speed of the robotic arm was $80 \mathrm{~mm} / \mathrm{sec}$, and standoff distance was $45 \mathrm{~mm}$. The overall procedure was almost is all details identical to the reported earlier (Ref 18, 19).

Two samples were produced using CS. 


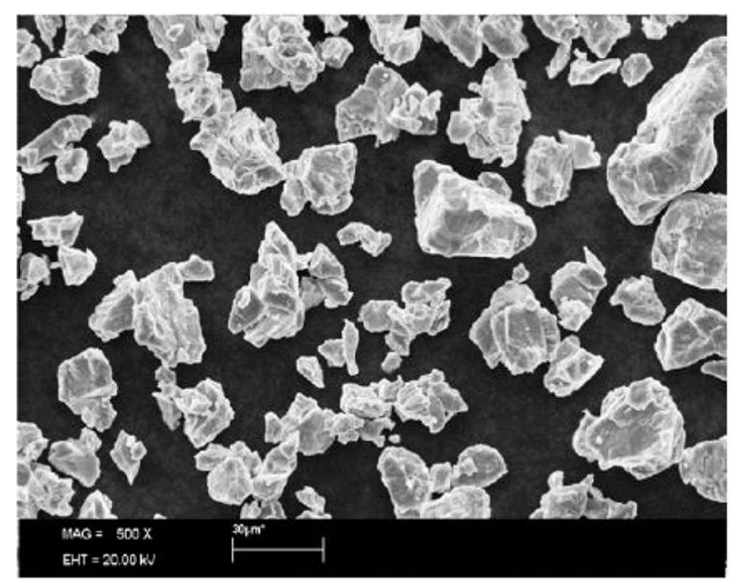

(a)

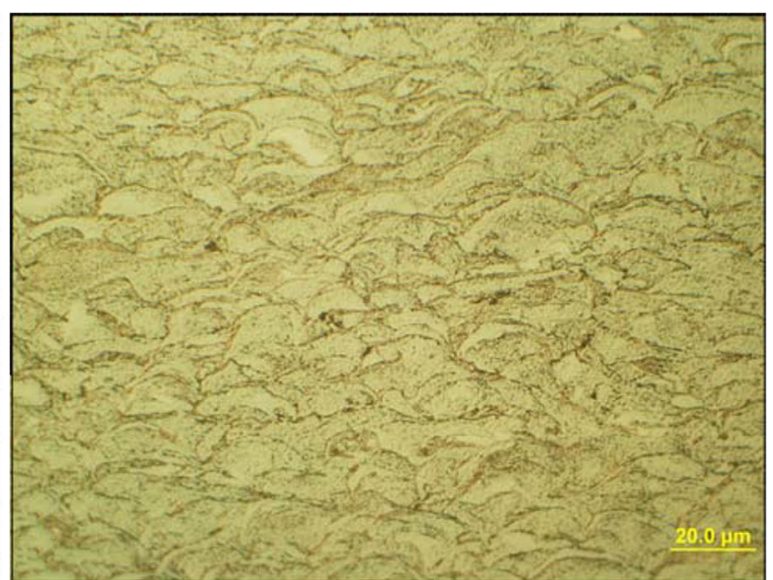

(b)

Fig. 1 (a) Scanning electron micrograph of the CP titanium powder used in this study and (b) microstructure of the cold-sprayed CP titanium

Fig. 2 Two CS samples in shapes of (a) coating and (b) bar

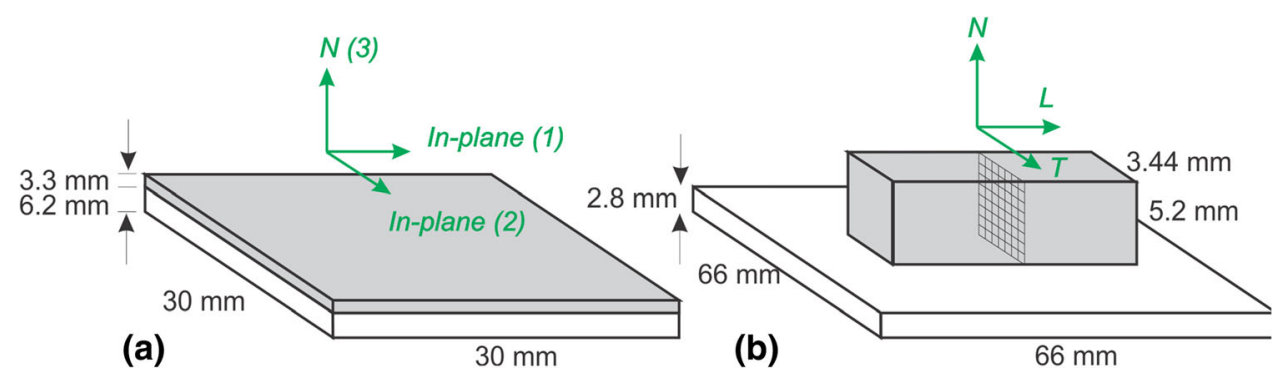

(b)

\section{Neutron Residual Stress Measurements Strategy}

With two different sample geometries and dimensions, the measurement strategies in the two cases were different and based on the following considerations regarding the expected stress state and required spatial resolution.

(1) The coating sample is a typical representative of a system that very accurately abides to the plane-stress condition. The applicability of the plane-stress condition in coated samples has been demonstrated practically but above that has a strong theoretical basis from the elasticity theory. First, the normal stress component (normal to the surface) is exact zero on both surfaces of the coated sample, $\sigma_{33}=0$. (Here, $x_{3}$ is the through-thickness dimension, while $x_{1}$ and $x_{2}$ are the in-plane coordinates.) Second, the condition for changing of $\sigma_{33}$ in the through-thickness direction is determined by the existence of the gradient of other stress components stated by the following equation of equilibrium for the normal component:

$\partial \sigma_{33} \partial x_{3}=-\left(\partial \sigma_{13} \partial x_{1}+\partial \sigma_{23} \partial x_{2}\right)$.

Thus, with uniform samples in $x_{1}$ and $x_{2}$ dimensions (no gradient), there must be no gradient of the $\sigma_{33}$ in the through-thickness direction. As a result, the plane-stress value of the Young's modulus was $68 \mathrm{GPa}$ (for solid Ti, the bulk value is $\sim 116 \mathrm{GPa}$ ) with statistical uncertainty less than $1 \%$. 
condition holds true through the whole thickness of a coated sample.

Of course, this condition breaks when location is close to the edge of a sample with large gradients in $x_{1}$ and $x_{2}$ dimensions.

Furthermore, when the spraying of particles is perpendicular to the surface, it is expected that symmetry of the process is reflected into the stress state of the sample. Thus, the normally expected stress state has the isotropic in-plane symmetry therefore the equal-biaxial stress state, $11=22$. This assumption is not valid anymore when spraying is done under certain tilt angle to the surface, and in this case, a biaxial stress state is rather expected, $11 \neq 22$. Therefore, depending on the exact production conditions, the residual stress state is characterized by a one-dimensional, throughthickness dependence of one or two in-plane stress components that should be reconstructed from the measured strains.

(2) The bar sample represents a more difficult case. In general, it is expected that stress field is $3 \mathrm{D}$, but limiting ourselves to a central transverse cross section (Fig. 2b) because its symmetry can provide some simplification. Three principal directions are the longitudinal (L), transverse (T) and normal (N) components, though locally (e.g., in the areas close to corners) they are not the principal directions anymore. For practical characterization, it is required to measure three normal components in the $2 \mathrm{D}$ cross section.

While these three components are allowed to have certain distributions from general elasticity theory point of view, due to large difference in dimensions some approximations might be contemplated. For example, $3.4 \mathrm{~mm}$ wall thickness can be considered as thin wall in comparison with longitudinal dimension of $40 \mathrm{~mm}$; therefore, the transverse stress component is expected to be very weak (and it is exactly zero on the vertical walls) in comparison with the longitudinal one. A similar consideration regarding the normal component can also suggest that this component is going to be weak. Essentially, if looking at the cross section, the stress field in the deposit is supposed to be one-dimensional with only dependence along wall height. Nevertheless, our measurement strategy did not involve any of these simplifying assumptions and three normal stress components were determined in the central $2 \mathrm{D}$ cross section in order to prove or disprove the above considerations.

The stress distribution in the Al baseplate central cross section is expected to be more complex with throughthickness and transverse dimension dependence. To measure this field in any full manner would require too much of neutron beamtime. Only one central line was measured for control purposes and, nevertheless, that provided sufficient additional information to characterize and validate our numerical stress modeling attempts.

\section{Experimental Procedure}

Neutron diffraction residual stresses measurements have been taken using the stress diffractometer KOWARI at the ANSTO OPAL research reactor (Ref 20). The instrument is equipped with $\mathrm{Si}(400)$ monochromator with changeable take-off angle that allows choosing a neutron wavelength accordingly to measured material.

(1) Coating sample. For through-thickness strain measurements in the middle part of the coating sample, a gauge volume with dimensions $0.5 \times 0.5 \times 20 \mathrm{~mm}^{3}$ was used. The use of the match-stick gauge volume elongated in the inplane dimension allows full utilization of the planar sample geometry for maximizing the overall volume of the scattering material while maintaining the $0.5 \mathrm{~mm}$ throughthickness resolution. With $5 \mathrm{~mm}^{3}$ overall gauge volume, the count rate is sufficiently high for determining strain with typical statistical uncertainty of $5 \times 10^{-5}$ within a reasonable measurement time. For materials like steel, it usually translates into $10 \mathrm{MPa}$ uncertainty of the stress values. The exposure times were $\sim 2$ minute per position for the measurements in the Fe substrate and $\sim 40$ minutes per point for the Ti coating. Such big discrepancy is due to big difference in the neutron scattering properties of $\mathrm{Fe}$ and Ti: diffraction intensity of $\mathrm{Ti}$ is approximately 10 time smaller, while background is 10 times larger. Additionally, the cold spray materials have significant effect of peak broadening that further decrease the overall accuracy of the strain measurements and increase the measurement time.

The strain measurements in the steel substrate were taken using $\gamma-\mathrm{Fe}(311)$ reflection with a neutron beam wavelength of $1.54 \AA$, while $\mathrm{Ti}(103)$ reflection and wavelength of $1.89 \AA$ were used for strain measurements in $\mathrm{Ti}$ coating. The choice of different wavelengths was stipulated by requirement of providing approximately $90^{\circ}$-geometry for the both reflections that investigated, i.e., $\gamma$ - and $\alpha$ $\mathrm{Fe}(211)$ with the diffraction angles being $90^{\circ}$ and $82^{\circ}$, respectively.

The strain measurements were taken in many throughthickness positions covering the entire sample thickness. The $0.5 \mathrm{~mm}$ spacing between points was chosen to be proportional to the overall thickness of $9.5 \mathrm{~mm}$ and gauge volume size of $0.5 \mathrm{~mm}$. The strain measurement locations were determined from surface intensity scans (entry curves) to accuracy better than $0.03 \mathrm{~mm}$.

Despite the prediction that equi-biaxial stress state was most likely expected, two in-plane directions and one normal direction were measured in order to reconstruct two in-plane stress principal components under the assumption of plane-stress condition as discussed above. 
A "substrate only" sample was also measured with the same measurement protocol to quantify the pre-existing stress distribution in the substrate material due to production. It was subsequently subtracted from the coated system stress data so that the stress represents residual stress that is generated by the cold spray process only.

(2) Bar sample. Stress investigations were performed using a wavelength of $1.665 \AA$ using take-off angle of $79^{\circ}$ that put the $\mathrm{Al}(311)$ reflection close to the optimal $90^{\circ}$ geometry, while $\mathrm{Ti}(103)$ reflection is at $\sim 80^{\circ}$. A cube-like gauge volume of dimensions $1 \times 1 \times 1 \mathrm{~mm}^{3}$ gauge volume was used to scan across central transverse cross section of the sprayed Ti bar with dimensions $3.4 \times 5.4 \mathrm{~mm}$ (width $\times$ height) as well as for through-thickness scan of the $\mathrm{Al}$ substrate of $2.8 \mathrm{~mm}$ thickness. All measurements were taken with fully submerged gauge volumes. Strain accuracy of $100 \mu$ strain was achieved against a data acquisition time of $\sim 10$ minutes per measurement point for $\mathrm{Al}$ and $\sim 40$ minutes per measurement point for Ti. Measurement locations were determined from surface entry curves to accuracy better than $0.05 \mathrm{~mm}$. Measurements of three principal directions were taken to reconstruct three principal stress components in assumption of the constant $\mathrm{d}_{0}$.

For stress calculations from the measured strain in all cases, the isotropic elastic diffraction constants were used. They were evaluated in accordance with the material and (hkl) indices of the reflection from the corresponding single crystal elastic constants using IsoDEC program (Ref 21). After the calculations of the stress components, they were checked to insure the fulfillment of the available stress balance and boundary conditions.

\section{Fitting Experimental Stress profiles with Analytical Models}

The experimental stress profiles and maps are often misleading and confusing. For example, it is common to find a statement that cold spray generates compressive residuals stress. Although this is usually correct for the most surface layer of deposited material, there might be some interior layers of cold spray that are under tension; thus, this statement requires more accurate formulation and interpretation. Second, the magnitude and distribution of the residual stress always depend on the dimension of the sample and, for example, coated samples sprayed to different thickness will demonstrate different stresses. Not only the dimensions of the substrate but also the substrate material (or more precisely, the elastic and thermal properties of the substrate) that also plays a role in formation of the residual stress profiles. For example, the Young's modulus of a substrate would be associated with amount of elastic constraint provided by the substrate in respect to the coating. Thus, the stress values in the experimental data represent a rather complex convolution of the substrate and coating material properties, dimensions and geometries with inherent parameters that are characteristic of the spray process and its truly attributes. The general approach to deconvolute different parameters of the elastic system is to model stress fields, analytically or through FEM, with explicit expression of the dimensional and material properties parameters. Due to complexity of the stress calculations for bodies of arbitrary shape, only an FEM approach can be recommended in general. However, in the case of our given simple sample geometries, a much easier analytical solution approach is attempted in the following ways.

(1) The coating sample was treated through use of the layer deposition model developed by Tsui \& Clyne (Ref 13). This approach was tested and applied multiple times to stress analysis of coatings made by different techniques (Ref 22, 23), including the cold spray coatings (Ref $12,24,25)$. It is based on the one-dimensional stress distribution analysis of the coating-substrate system, which allows an analytical solution. This model gives an empirical description of the coating-substrate system with only two fitting parameters (while assuming that all thermal, elastic and dimensional properties of the coating and substrate are known):

(a) The deposition stress $\sigma_{\mathrm{d}}$. It is characteristic of the spray process; its sign and magnitude are determined by the spray process physical parameters (the main ones are particle temperature and velocity) as well as plastic properties of the sprayed material; it can be tensile (quenching) or compressive (peening).

(b) The thermal mismatch $\Delta \varepsilon_{\text {th. }}$. It accounts for the discrepancy in the thermal expansion coefficient (CTE) between materials of substrate and coating, $\Delta \varepsilon_{\text {th }}=\Delta \alpha \Delta \mathrm{T}$, where $\Delta \mathrm{T}$ is the temperature drop by at the end spraying process, while $\Delta \alpha$ is a difference in CTE. If the temperature is well monitored (known $\Delta \mathrm{T}$ ) and the materials CTE's are also known (then known $\Delta \alpha$ ), this parameter can be also fixed reducing the whole problem to one-parameter fitting.

Using this parametrization, the experimental throughthickness profiles can be fitted and these two parameters, $\sigma_{\mathrm{d}}$ and $\Delta \varepsilon_{\mathrm{th}}$, can be extracted. Based on this approach, separate contribution into the final stress state can be quantified and then factorized.

(2) The bar sample poses more complex elasticity theory problem with potentially three nonzero stress components and 2D stress distribution in the sample cross section. However, with certain simplification, the same approach based on the same parametrization can be applied. We can consider the same two parameters: 
(a) The deposition stress $\sigma_{d}$ to characterize the spray process, and

(b) The thermal mismatch $\Delta \varepsilon_{\text {th }}$ to accounts for the thermo-elasticity part if CTE's of the coating and substrate materials are different.

However, the deposition model must be reassessed. While the layer deposition model deals with the case of a free-standing substrate, which is allowed to bend and deform during the deposition process, the more appropriate model for the additive manufacturing of a thin wall (or bar) is to consider a fixed substrate on which the wall was is deposited and only then the substrate bending constraints are released. This kind of scenario was considered and quantitatively validated on a similar additively manufactured thin-wall sample made by SLM (selective laser melting) (Ref 26). Assuming the wall to be thin eliminates the necessity of the transverse component to be analyzedit should be very close to zero across the wall. However, this approximation depends on exact dimensional characteristics and at least requires an experimental verification, which has been done in this study.

Performing the above analysis allows parametrization and separation of different aspects of the CS process in its connection to the observed residual stress distribution. Also, the geometrical factors, such as thickness or material of substrate, can be eliminated and stress distribution can be reduced to few (one or two) parameters fully characterizing the elastic system and its stress field. In our case, those characteristic parameters, when extracted, allow to compare the deposition process for two samples despite their different geometries and dimensions. Furthermore, based on this parametrization approach, some prediction or recalculation of stress field can be made to the systems of wider class of sample geometries.

\section{Results}

(1) Coating sample. The results of through-thickness measurements of the two orthogonal directions are shown in Fig. 3 that demonstrates that the stress state of the sample is truly equi-biaxial, as was anticipated. Due to this, the two components were averaged and the averaged experimental profile was fitted with the model discussed in section 2.4 and this treatment resulted in a good agreement demonstrated in Fig. 4.

As discussed in section 2.4, the two fitting parameters were the deposition stress $\sigma_{\mathrm{d}}$ and the thermal mismatch $\Delta \varepsilon_{\mathrm{th}}$, whose numerical values are $\sigma_{\mathrm{d}}=-15 \pm 17 \mathrm{MPa}$ and the thermal mismatch $\Delta \varepsilon_{\mathrm{th}}=2000 \pm 200 \mu$ strain. This thermal mismatch value corresponds to a temperature drop

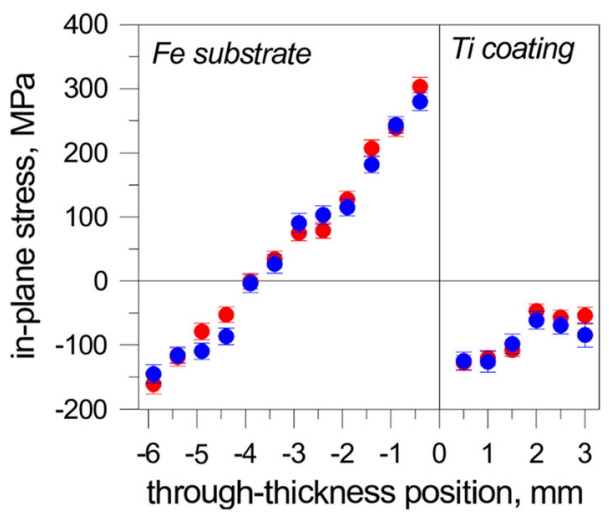

Fig. 3 Experimental check of residual stress equal-biaxiality for the cold-sprayed $\mathrm{Ti} / \mathrm{Fe}$ coated sample (Fe stands for stainless steel here). Two orthogonal stress components (red and blue) were measured independently

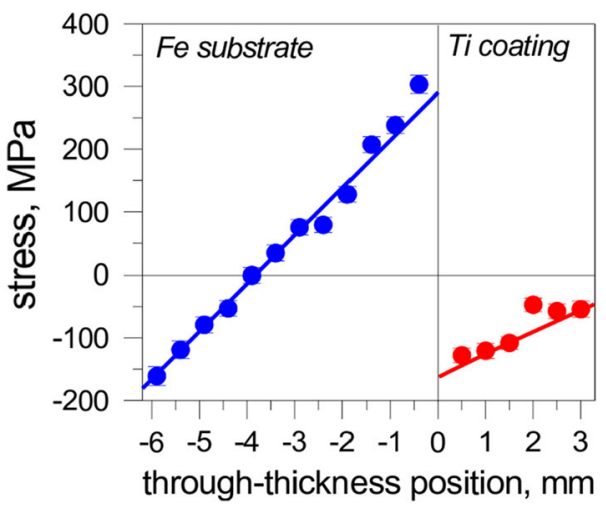

Fig. 4 Through-thickness stress distribution in the cold-sprayed Ti/ Fe coated sample (Fe stands for stainless steel here). The experimental data (symbols) are fitted with a model (lines)

of $\Delta \mathrm{T} \sim 250^{\circ} \mathrm{C}$, evaluated from the known CTE of steel and Ti.

For the purpose of the numerical comparison of the two terms to the total stress profile, the decomposition of the overall stress profile is shown in Fig. 5. The figure clearly demonstrates that the total stress results mostly from the thermal mismatch between Fe substrate and Ti coating, while the deposition stress has a magnitude approximately 10 times smaller.

(2) The bar sample. The experimental stress maps of the three orthogonal components in the $\mathrm{Ti}$ deposit part are shown in Fig. 6. The maps interpretation must take into account that the experimental uncertainties are $\sim 25 \mathrm{MPa}$. Having this in mind, is should be accepted that the transverse and normal components have not demonstrated any statistically significant dependence and can be approximated as zeros (a much more accurate measurement is required to see possible effects for the two components). In contrast, the longitudinal component showed clear variation as a function of the height, while through-wall 
Fig. 5 Decomposition of the stress profile into two contributions, the thermal mismatch stress (first term) and the deposition stress (second term). (Fe stands for stainless steel here.) Note the change of the scale (factor of 10) for the second term

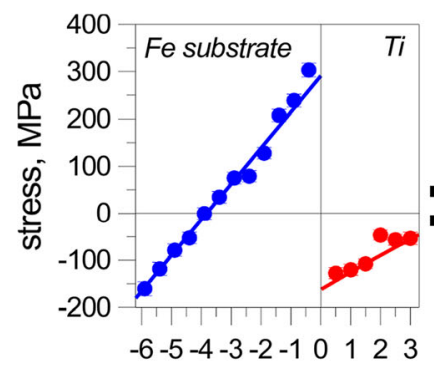

through-thickness position, $\mathrm{mm}$

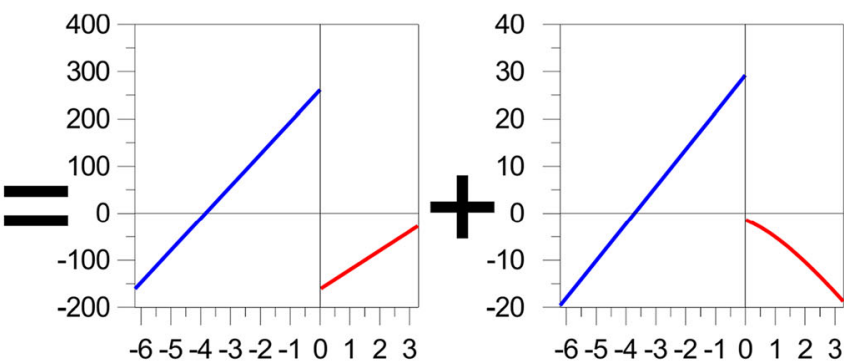

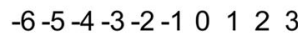
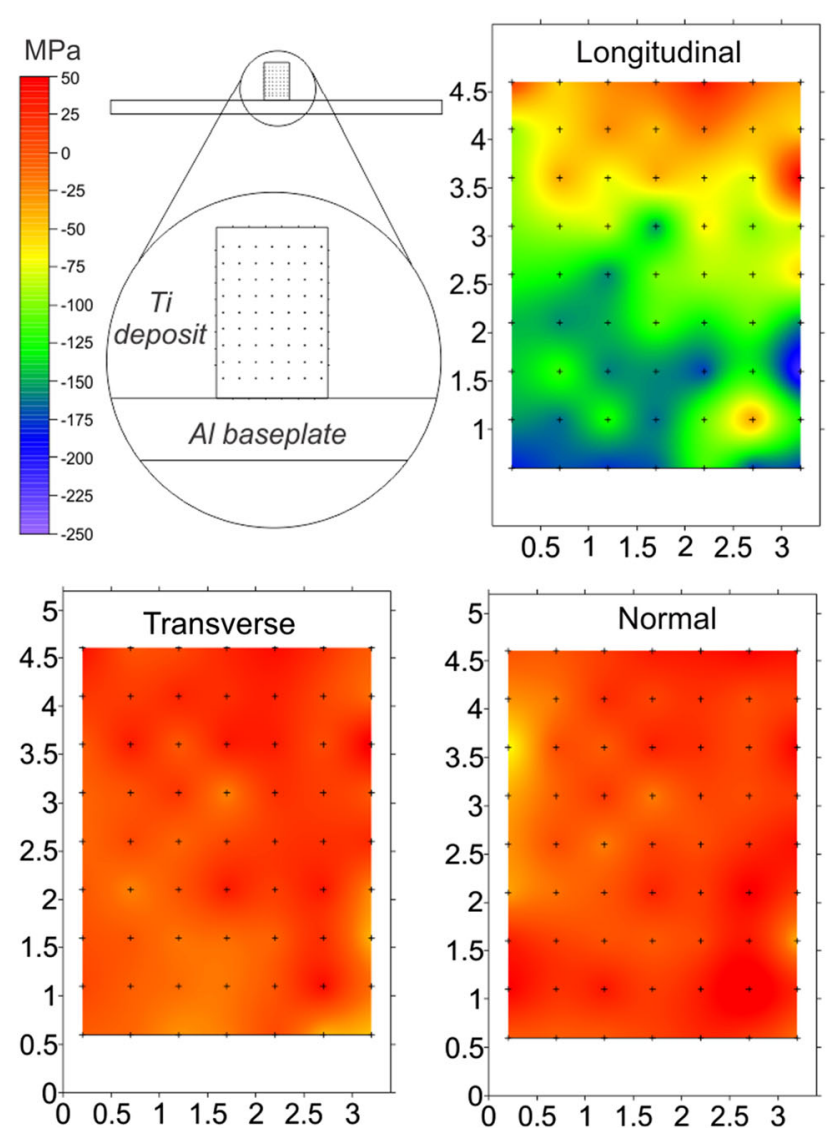

Fig. 6 Experimental maps of three stress components measured in the bar sample central cross section

variation did not exhibit any statistically meaningful dependence.

Thus, based on the statistical analysis of 2D stress maps data, the whole dataset was reduced to a single longitudinal component through-height dependence shown in Fig. 7, where the through-thickness profile on $\mathrm{Al}$ substrate is also added. The reduced 1D stress profile was also treated within the model approach described in chapter 2.4, and results of fitting the model into the experimental data are shown in the same figure as well. The same way as for the coated sample, the relative contributions of the two fitting model parameters / terms are shown in Fig. 8, where corresponding numerical values are $\Delta \varepsilon_{\text {th }}=2200 \pm 250 \mu$ strain

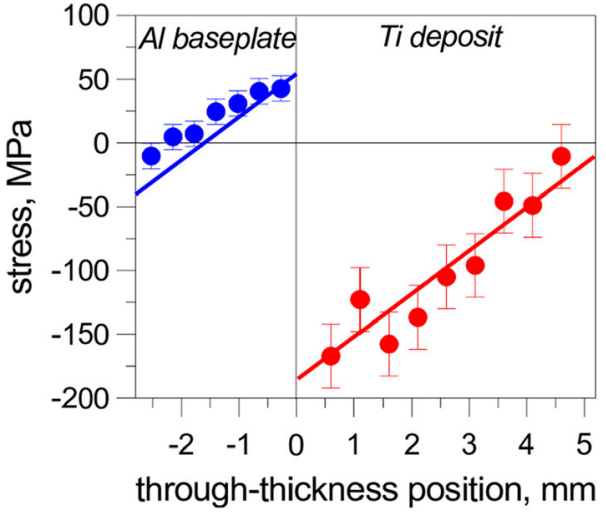

Fig. 7 Through-thickness stress distribution in the cold-sprayed Ti/ Fe coated sample. The experimental data (symbols) is shown in comparison with a model (lines)

and $\sigma_{\mathrm{d}}=-20 \mathrm{MPa}$. With known CTE of $\mathrm{Al}$ and $\mathrm{Ti}$, the temperature drop can also be evaluated as $\Delta \mathrm{T} \sim 150^{\circ} \mathrm{C}$.

The same way as for the coated sample, mutual comparison of the two terms and contribution into the total stress profile is illustrated in Fig. 8. Similarly to the case of the coated sample, the dominating term here is the thermal mismatch (due to the CTE difference between $\mathrm{Al}$ substrate and $\mathrm{Ti}$ deposit), with contribution from the deposition stress approximately 10 times smaller. The fact that in both cases the thermal mismatch is 10 times greater than the deposition stress is rather a coincidence.

\section{Discussion}

With two given generic sample geometries, the results suggest that in terms of residual stress development, an attention must be paid on matching the CTE of the deposited material and the substrate material. With inevitable elevated spraying temperature $(\Delta \mathrm{T}$ in the range of $100^{\circ} \mathrm{C}$ to $250^{\circ} \mathrm{C}$ ), any significant difference in CTE, say $\Delta \alpha \sim 5 \times 10^{-6}$ (as in case of $\mathrm{Ti}$ and $\mathrm{Fe}$ or $\mathrm{Ti}$ and $\mathrm{Al}$ ) can result in a thermal mismatch stress with magnitude of several hundred MPa. In our case, the thermal mismatch by chance was approximately the same for the both samples, though the temperature drop was somewhat different, $\Delta \mathrm{T}$ 
Fig. 8 Decomposition of the stress profile into two contributions, the thermal mismatch stress (first term) and the deposition stress (second term). Note the change of the scale (factor of 10) for the second term

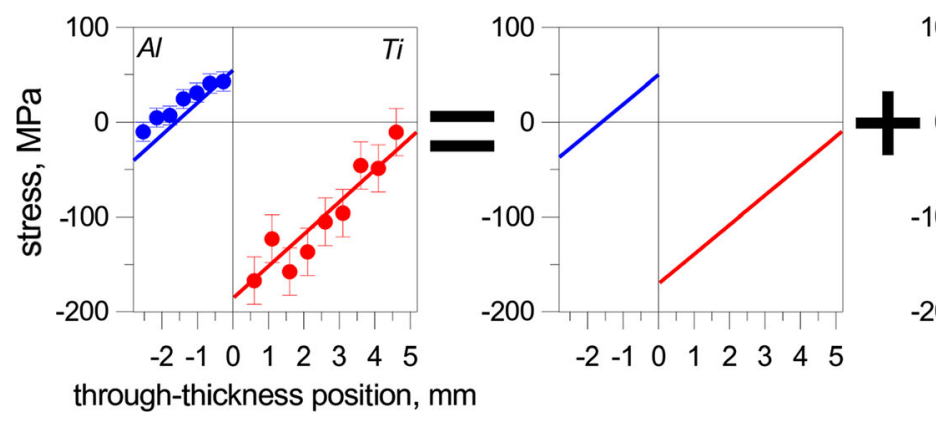

through-thickness position, $\mathrm{mm}$

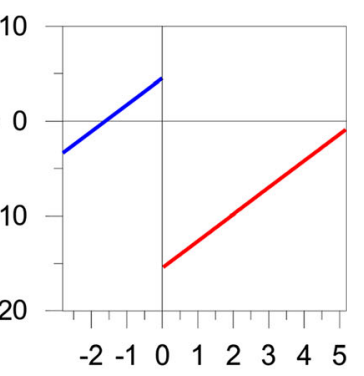

$\sim 250^{\circ} \mathrm{C}$ for the coated sample and $\Delta \mathrm{T} \sim 150^{\circ} \mathrm{C}$ for the bar sample. The difference in temperature conditions is most evidently due to the presence of the mask during deposition of the bar sample that played the role of a heat shield and reduced amount of the hot gas coming to the sample.

The overall stress profiles for both studied samples are dominated by the thermal mismatch stress, which in its effect is about one order of magnitude larger than the effect from the deposition stress, the second source of the residual stress. Since thermal mismatch for both studied systems has liner stress response and distribution, this was observed experimentally and can be match with the analytical approach.

This thermal mismatch stress can be easily eliminated by a choice of the substrate/baseplate with the same CTE as the CS powder. Also, this term can be eliminated by cutting off the substrates, as almost all AM samples are assumed to be removed from the baseplate, but until this can be done the accumulated stresses might have already develop some cracking in the sample; thus, this approach might not be always reliable. In addition to that, it should be taken into account that inappropriate cutting methods can case yet another contribution to the overall residual stress field. Mechanical cutting is known to produce a tensile residual stress on the cut surface, and this method should be avoided. The best practice in AM techniques such as SLM is the EDM wire cutting (electric discharge machining) that reduces to minimum such negative impact.

In conditions of this study on $\mathrm{CP}$ titanium, the $\mathrm{CS}$ process by itself generates very mild compressive deposition stress, approximately - $20 \mathrm{MPa}$. A similar deposition stress value of $-15 \mathrm{MPa}$ was also obtained in Ti coatings sprayed in slightly different spraying conditions (Ref 12). Compressive stress of a moderate magnitude on surface ${ }^{1}$ is

\footnotetext{
1 "Compressive stress on surface" mentioned above is technically incorrect use of term because there is no stress on surface (or interface for that matter), and it is always "compressive stress in the infinitesimal most surface layer of material" because stress is a bulk quantity. However, this "jargon" term is used below for the sake of tradition (coming from the surface x-ray measurement) and compactness.
}

considered to be beneficial because compressive stress does not induce crack formation or crack propagating even though some localized defects can be present on the surface. At the same time, mild compressive stress on surface inevitable must be balanced by tensile stress in the inner parts of an AM part. However, when compressive surface stress is mild the balancing tensile stress inside is most likely to be of lesser absolute magnitude and, unless some inner defects are present, cannot be considered as bigger risk factor in comparison with tensile stress on surface. Therefore, potentially CS is a suitable candidate for AM technique from the residual stress point of view. However, CS can induce tensile deposition (and/or surface) stress. This happens when for the purpose of more efficient deposition of the plastically hard material the spraying temperature is too high. One example of the CS operating in the thermal spray regime is Ti sprayed at $1100{ }^{\circ} \mathrm{C}$ and 50 bar pressure that demonstrate tensile (quenching) deposition stress (Ref 27). Another example of CS system that generated tensile (quenching) stress is high-strength duplex stainless steel that was sprayed by CGT KINETICS 4000 at $800{ }^{\circ} \mathrm{C}$ and 35 bar pressure. Because tensile stress facilitates development of the surface cracks, the CS regimes that generate tensile deposition stress are not desirable from the structural integrity viewpoint.

For the given two samples fabricated with a unidirectional spraying perpendicular to the surface, the micromechanical stress formation mechanisms were the same leading to almost identical deposition stress. The geometry factor led to the fact that these two generic sample geometries formed different stress states on the surface, the equal-biaxial in one case and uniaxial in the other. Although these two stress states are simple and one-dimensional, the results can be generalized for some arbitrary geometries and shapes that can potentially be produced by CSAM. When operating in the AM mode, it is expected that the surface stresses are to be approximately of the value of the deposition stress if we consider any locally flat piece of surface or less if we consider area close to sample edges. This conclusion holds almost the same when we consider unidirectional deposition or deposition under different angles in respect to the sample coordinate system. 
In comparison, other AM techniques like SLM can produce stresses with several hundreds of MPa with tensile stress on the surface (Ref 28) that might cause cracking and distortions during deposition or requires certain stress relief heat treatment to eliminate large residual stresses.

\section{Summary and Conclusions}

Two deposits of CP titanium of different geometries were manufactured by cold spray. One sample was a planar thick coating, while the other was a rather thick wall deposit (thick in comparison with the wall height) on the top of baseplate. The residual stress states of the two samples were experimentally determined by the neutron diffraction technique, and despite difficulties of measuring titanium, the resultant accuracy was sufficient to analyze the experimental results quantitatively applying relevant parametric models. It was demonstrated that in the both cases the residual stress was mainly formed through thermal mismatch mechanism, due to the CTE difference between substrate (baseplate) and deposited CT titanium. In both cases (coincidently), this effect was approximately 10 times larger than the deposition stress, which was slightly compressive, approximately - $20 \mathrm{MPa}$. The former can be eliminated by selecting substrate (baseplate) material with the same CTE (same material) or simply cutting off the substrate (baseplate). The latter, the deposition stress, cannot be eliminated by selection of the substrate material; thus, it is to be in the focus of AM technology. The magnitude and the sign of the deposition stress, - $20 \mathrm{MPa}$, are determined by the spraying conditions and the CS system used in this study, but can be changed by varying the temperature-pressure parameters of the CS process.

Although two simple sample geometries were studied, the results demonstrate the dependence of the sample geometry on the residual stress state. While the compressive stress accumulated on the surface in conjunction with a zone of tensile stress in the inner volume of a sample is a common feature in the both cases, a change in the sample geometry leads to the transition in the stress state from equal-biaxial to uniaxial .

CSAM of this study demonstrated that it is potentially an advantageous technique from the point of view of stress analysis. It forms slightly compressive stress on surface ( $-20 \mathrm{MP}$ in this study for $\mathrm{Ti}$ ) which is generally beneficial because of its ability to prevent crack formation and crack propagation and build up much lesser magnitude of the residual stress in comparison with other AM techniques, e.g., SLM. Also, while in our study the deposition stress (surface layer generated stress) is mildly compressive, there are some regimes when CS can produce tensile deposition stress that should be avoided.
Acknowledgement This work was also supported by the Australian Nuclear Science and Technology Organisation (ANSTO) through proposals P1211 and P596.

\section{References}

1. R. Huang and H. Fukanuma, Future trends in cold spray techniques, 6-Future Development of Thermal Spray Coatings, N. Espallargas, Ed., Woodhead Publishing, Sawston, 2015, p 143162

2. S. Yin, B. Aldwell, and R. Lupoi, Cold spray additive manufacture and component restoration, Cold-Spray Coatings: Recent Trends and Future Perspectives, P. Cavaliere, Ed., Springer International Publishing, Cham, 2018, p 195-224

3. S. Yin, P. Cavaliere, B. Aldwell, R. Jenkins, H. Liao, W. Li, and R. Lupoi, Cold Spray Additive Manufacturing and Repair: Fundamentals and Applications, Addit. Manuf., 2018, 21, p 628-650

4. R.N. Raoelison, C. Verdy, and H. Liao, Cold Gas Dynamic Spray Additive Manufacturing Today: Deposit Possibilities, Technological Solutions and Viable Applications, Mater. Des., 2017, 133, p 266-287

5. H. Assadi, F. Gärtner, T. Stoltenhoff, and H. Kreye, Bonding Mechanism in Cold Gas Spraying, Acta Mater., 2003, 51(15), p 4379-4394

6. M. Grujicic, J.R. Saylor, D.E. Beasley, W.S. DeRosset, and D. Helfritch, Computational Analysis of the Interfacial Bonding Between Feed-Powder Particles and The Substrate in The ColdGas Dynamic-Spray Process, Appl. Surf. Sci., 2003, 219(3-4), p 211-227

7. M. Grujicic, C.L. Zhao, W.S. DeRosset, and D. Helfritch, Adiabatic Shear Instability Based Mechanism for Particles/Substrate Bonding in the Cold-Gas Dynamic-Spray Process, Mat. Des., 2004, 25(8), p 681-688

8. W.Y. Li, C. Zhang, C.J. Li, and H. Liao, Modeling Aspects of High Velocity Impact of Particles in Cold Spraying by Explicit Finite Element Analysis, J. Therm. Spray Technol., 2009, 18(5-6), p 921-933

9. R. Ghelichi, S. Bagherifard, M. Guagliano, and M. Verani, Numerical Simulation of Cold Spray Coating, Surf. Coat. Technol., 2011, 205(23-24), p 5294-5301

10. W. Li, K. Yang, D. Zhang, and X. Zhou, Residual Stress Analysis of Cold-Sprayed Copper Coatings by Numerical Simulation, $J$. Therm. Spray Technol., 2016, 25(1), p 131-142

11. M. Saleh, V. Luzin, and K. Spencer, Analysis of the Residual Stress and Bonding Mechanism in the Cold Spray Technique Using Experimental and Numerical Methods, Surf. Coat. Technol., 2014, 252, p 15-28

12. V. Luzin, K. Spencer, M. Zhang, N. Matthews, J. Davis, and M. Saleh, Residual Stresses in Cold Spray Coatings, Cold-spray Coatings: Recent Trends and Future Perspectives, P. Cavaliere, Ed., Springer International Publishing, Cham, 2018, p 451-480

13. Y.C. Tsui and T.W. Clyne, An Analytical Model for Predicting Residual Stresses in Progressively Deposited Coatings Part 1. Planar Geometry, Thin Solid Films, 1997, 306(1), p 23-33

14. Y.C. Tsui and T.W. Clyne, An Analytical Model for Predicting Residual-Stresses in Progressively Deposited Coatings Part 2. Cylindrical Geometry, Thin Solid Films, 1997, 306(1), p 34-51

15. A. Papyrin, Cold Spray Technology, Adv. Mater. Process., 2001, 159(9), p 49-51

16. G.G. Stoney, The Tension of Metallic Films Deposited by Electrolysis, Proc. R. Soc. London, Ser. A , 1909, 82(553), p 172175 
17. Y.C. Tsui, S.C. Gill, and T.W. Clyne, Simulation of the Effect of Creep on Stress Fields During Vacuum Plasma Spraying onto Titanium Substrates, Surf. Coat. Technol., 1994, 64(2), p 61-68

18. S.H. Zahiri, C.I. Antonio, and M. Jahedi, Elimination of Porosity in Directly Fabricated Titanium via Cold Gas Dynamic Spraying, J. Mater. Process. Technol., 2009, 209(2), p 922-929

19. S.H. Zahiri, D. Fraser, and M. Jahedi, Recrystallization of Cold Spray-Fabricated CP Titanium Structures, J. Therm. Spray Technol., 2009, 18(1), p 16-22

20. O. Kirstein, V. Luzin, and U. Garbe, The Strain-Scanning Diffractometer Kowari, Neutron News, 2009, 20(4), p 34-36

21. T. Gnaupel-Herold, ISODEC: Software for Calculating Diffraction Elastic Constants, J. Appl. Crystallogr., 2012, 45(3), p 573574

22. V. Luzin, A. Valarezo, and S. Sampath, Through-Thickness Residual Stress Measurement in Metal and Ceramic Spray Coatings by Neutron Diffraction, Mater. Sci. Forum, 2008, 571-572, p 315-320

23. V. Luzin, A. Vackel, A. Valarezo, and S. Sampath, Neutron Through-Thickness Stress Measurements in Coatings with High Spatial Resolution, Mater. Sci. Forum, 2017, 905, p 165-173
24. V. Luzin, K. Spencer, and M.X. Zhang, Residual Stress and Thermo-Mechanical Properties of Cold Spray Metal Coatings, Acta Mater., 2011, 59(3), p 1259-1270

25. K. Spencer, V. Luzin, N. Matthews, and M.X. Zhang, Residual Stresses in Cold Spray Al coatings: The Effect of Alloying and of Process Parameters, Surf. Coat. Technol., 2012, 206(19-20), p 4249-4255

26. V. Luzin and N. Hoye, Stress in Thin Wall Structures Made by Layer Additive Manufacturing, Mater. Res. Proc., 2016, 2, p 497502

27. D. Boruah, B. Ahmad, T.L. Lee, S. Kabra, A.K. Syed, P. McNutt, M. Doré, and X. Zhang, Evaluation of Residual Stresses Induced by Cold Spraying of Ti-6Al-4V on Ti-6Al-4V Substrates, Surf. Coat. Technol., 2019, 374, p 591-602

28. T. Gnäupel-Herold, J. Slotwinski, and S. Moylan, Neutron Measurements of Stresses in a Test Artifact Produced by LaserBased Additive Manufacturing, AIP Conf Proc, 2014, 1581(1), p 1205-1212

Publisher's Note Springer Nature remains neutral with regard to jurisdictional claims in published maps and institutional affiliations. 